\title{
Retinal thickness variation in the diabetic patient measured by the retinal thickness analyser
}

\author{
Dov Weinberger, Ruth Axer-Siegel, David Landau, Yuval Yassur
}

\begin{abstract}
Aim-To evaluate the potential of the retinal thickness analyser (RTA) as an objective tool for assessment and follow up of diabetic macular oedema.

Methods-A prototype of the RTA that operates on the principle of laser slit biomicroscopy was used. Retinal thickness was obtained in 41 eyes of 41 diabetic patients. The clinical diagnosis was cystoid macular oedema (CMO) in 10 eyes, clinically significant macular oedema (CSMO) without retinal cysts in 21 eyes, and "dry" macula following grid pattern laser treatment in 10 eyes. The control group consisted of 46 eyes of age matched healthy volunteers.
\end{abstract}

Results-In normal eyes (46 eyes), the foveal thickness measured was 178 (SD 44) $\mu \mathrm{m}$ and the macular thickness around the fovea was 311 (51) $\mu \mathrm{m}$. The eyes with CMO displayed the largest foveal thickening, 875 (287) $\mu \mathrm{m}$ (390\% increase compared with normal values). The average thickness of the fovea in the non-cystoid CSMO group was 427 (175) $\mu \mathrm{m}$ (144\% increase compared with normal fovea). The average thickness of the foveal centre in eyes judged as having "dry" macula after laser treatment was 315 (71) $\mu \mathrm{m}$ (77\% increase compared with normal value and a $26 \%$ decrease in thickness compared with the CSMO eyes). Statistically significant differences were found in central thickness between these four groups $(p=0.0001)$. The average thickness at $500 \mu \mathrm{m}$ surrounding the fovea was $566(202) \mu \mathrm{m}$ in the CSMO eyes compared with 311 (51) $\mu \mathrm{m}$ in normal eyes ( $80 \%$ increase). The "dry" macula group (after undergoing laser treatments) had an average thickness of 414 (94) $\mu \mathrm{m}$ (27\% decrease compared with CSMO eyes and a $33 \%$ increase compared with eyes of healthy controls).

Conclusions-RTA is a system for quantifying macular thickness and imaging of macular pathology. The system can be a useful tool for diagnosis of macular diseases and for evaluation of the effect of treatment modalities.

(Br F Ophthalmol 1998;82:1003-1006)

Optical imaging instruments of ocular structures have been developed in recent years with many clinical applications. These techniques are non-invasive and enhance information concerning surface and internal structures of the ocular tissue.
Retinal thickness analysis is of great importance in understanding pathophysiology and treatment efficacy in many macular diseases, including diseases in which the retina becomes thicker, such as cystoid macular oedema, diabetic macular oedema, or diseases in which the retinal tissue becomes thinner or is missing (macular hole, cystoid macular oedema, and scarred area after laser treatment).

The assessment of retinal thickening or thinning by slit lamp biomicroscopy or stereo fundus photography is often difficult, very subjective, and of questionable reliability. Moreover, these methods necessitate the time consuming involvement of a highly skilled observer.

A quantitative method of measuring retinal thickness and visualisation of fine retinal structures has already been introduced by Zeimer $e t$ $a l,{ }^{1}$ Shahidi et $a l,{ }^{2}$ and Mori et $a l^{\beta}$; this method is based on angular delivery of a narrow, green, helium neon laser beam onto the retina and detection of the intersection of the slit with the retinal structures.

Recently, an instrument called the retinal thickness analyser (RTA, Talia Technology Ltd, Mevaseret Zion, Israel), based on the same method has been introduced by Zeimer et $a l .{ }^{4}$ The instrument is able to scan and measure the thickness of a selected retinal area in various regions of the fundus, including the macula. ${ }^{5}$ Landau et $a l^{5}$ reported the efficacy and accuracy of this instrument in quantitative retinal thickness measurements in healthy subjects.

We hereby present the results of retinal thickness measurements by the RTA in the macular area in a normal age matched group of patients compared with diabetic patients before and after laser treatment.

\section{Patients and methods}

PRINCIPLE OF THE RTA

The RTA operates on the principle of slit lamp biomicroscopy. A narrow $(15 \mu \mathrm{m})$ slit of green helium neon light (543 nm), $2 \mathrm{~mm}$ long, is projected onto the retina at an angle of 15 degrees. The light scattered back from the retina is viewed by a RTA TV camera at an angle opposite to the angle of the incidence light. Concomitantly, a second fundus TV camera shows the fundus image for orientation of the slit. Scanning of 10 adjacent cross sections of the retina, about $200 \mu \mathrm{m}$ apart, covering a total area of approximately $2 \times 2 \mathrm{~mm}$, lasts only $200 \mathrm{~ms}$. Nine adjacent squares can be scanned which cover a zone of about $6 \times 6$ $\mathrm{mm}\left(20^{\circ} \times 20^{\circ}\right)$ around the fovea. The intersection of the laser slit with the anterior 


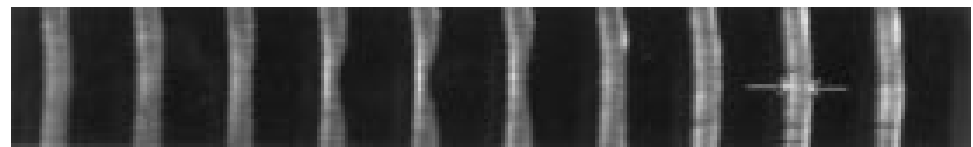

Figure 1 Ten adjacent optical cross sections of the retina covering an area of about $2 \times 2$ $\mathrm{mm}$. The separation of the white lines is proportional to the thickness of the retina at that location. In these images the inner limiting membrane is on the right (right arrow) and the retinal pigment epithelium is on the left (left arrow).

and posterior surfaces of the retina constitute the anterior and posterior border of the image of the cross section of the retina. The distance of the white lines between the arrows is proportional to the thickness of the retina at that location. In these images the inner limiting membrane is represented by the right arrow and the retinal pigment epithelium (RPE) by the left arrow. The image is acquired within $200 \mathrm{~ms}$ for scanning of a square area of $2 \times 2$ $\mathrm{mm}$ (which is faster than the saccadic eye movement). Measurements are performed at 10 points on each of 10 slits which cover a total area of $2 \times 2 \mathrm{~mm}$. Nine such areas are scanned for mapping of the posterior pole, covering a 6 $\times 6 \mathrm{~mm}$ area with 900 measurement points. At each measurement point, the line profile, which represents the retinal thickness, is determined by averaging the measured pixels. Each 10 pixels represent $25 \mu \mathrm{m}$ on the retina.

Visualisation of fine structures provides the capability to acquire 10 slit images covering a small area in a very short period of time, and display of these images side by side allows for better visualisation of fine details.

Green light is strongly absorbed by haemoglobin and this causes a very sharp definition of the RPE side of the retinal cross section compared with the slit image obtained with other wavelengths and because of this, no light scatters back from tissue behind the RPE.

The laser output is $110 \mu \mathrm{W}$ into a slit of about $2 \mathrm{~mm}$ length and $15 \mu \mathrm{m}$ width.

The image is processed by computer software and the output may be displayed as 10 adjacent cross sections of the retina (Fig 1), as a topographic map (Fig 2), or as a numeric report.

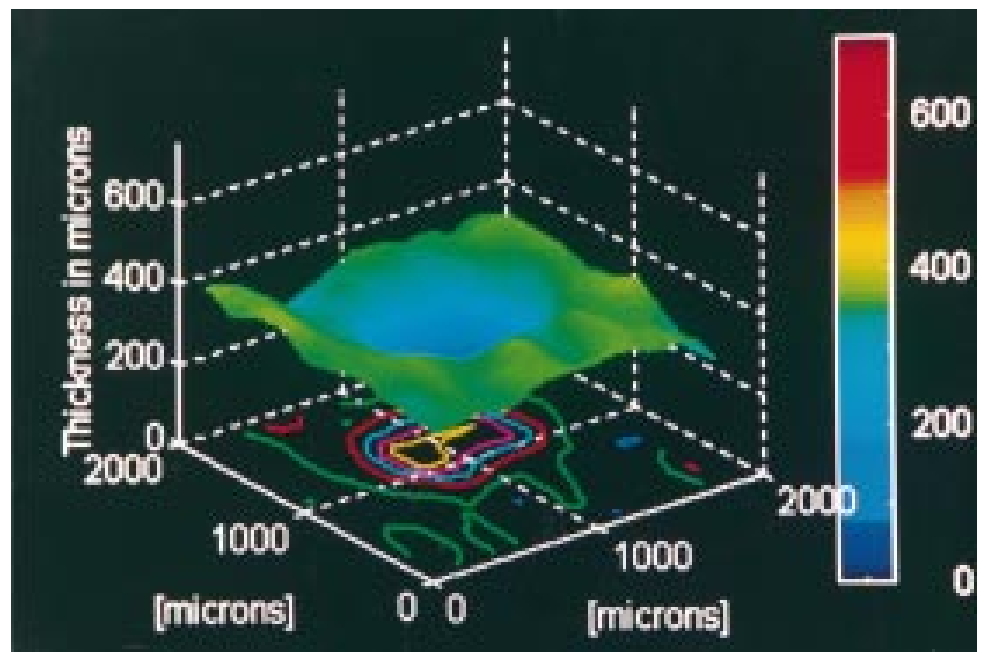

Figure 2 Three dimensional topographic presentation of the retinal thickness an area of the fovea, $2 \times 2 \mathrm{~mm}$, of a normal patient. The coloured scale represents the thickness in microns $(\mu m)$ and the map represents the variation in thickness in and around the fovea.
The reproducibility of the instrument was assessed by measuring the foveal thickness of 10 normal eyes. The intravisit reproducibility evaluated nine scans centred on the fovea in a single session and the intervisit reproducibility evaluated two scans of the foveal centre measured in visits 2 days apart. One hundred thickness points were measured on each scan and the coefficient of variance (SD/mean) was calculated for each of the 100 measured spots.

\section{PATIENT DATA}

We measured the retinal thickness in three groups of eyes of diabetic patients. Before the measurement, informed consent was obtained from all patients and healthy controls. The clinical diagnosis of these patients was determined by biomicroscopy according to early treatment diabetic retinopathy study (ETDRS) criteria $^{6}$ and by fluorescein angiography. The first group comprised 10 eyes with cystoid macular oedema (CMO), the second group consisted of 21 eyes with clinically significant macular oedema (CSMO) without retinal cysts, and the third group included 10 eyes which were judged as having "dry" macula following previous grid pattern laser treatment due to CSMO. The results of the retinal thickness were compared with those of 46 normal eyes of age matched healthy controls. The data of the normal individuals have already been published, ${ }^{5}$ including the reproducibility data. The pupils were dilated with $1 \%$ tropicamide and $2.5 \%$ phenylephrine hydrochloride. An area of $6 \times 6 \mathrm{~mm}$, centred at the fovea, was scanned. In order to compare the retinal thickness between the various groups of eyes, the measurements at the centre of the fovea as well as four squared area of $2 \times 2 \mathrm{~mm}$ located at 12 , 3,6 , and 9 o'clock on a circle, with a radius of $500 \mu \mathrm{m}$ centred on the fovea, were calculated and averaged.

In order to compare the retinal thickness between the four groups, analysis of variance was performed, using the multiple comparison options of Dunnet and Duncan. Analysis of variance was also done specifically for three age groups (20-34, 35-49, and 50-70 years). Probability values less or equal to 0.05 were considered statistically significant.

\section{Results}

The intravisit reproducibility of nine scans evaluated the centre of the fovea in a single session, obtained on the same day, was plus or minus $5.9 \%$, corresponding to plus or minus $10.6 \mu \mathrm{m}$. The intervisit reproducibility of two scans evaluated the centre of the fovea, obtained on visits 2 days apart, was plus or minus $6.6 \%$, corresponding to plus or minus $10.8 \mu \mathrm{m}$.

The macular thickness measurements in the various groups are displayed in Table 1 . The thickness of the fovea in the CSMO eyes ranged between $200 \mu \mathrm{m}$ and $757 \mu \mathrm{m}$, with an average of 427 (SD 175) $\mu \mathrm{m}$ compared with a range of $100-255 \mu \mathrm{m}$, with an average of 178 (44) $\mu \mathrm{m}$ in normal eyes, a $144 \%$ increase. The thickness range of the foveal centre in eyes judged as having "dry" macula after laser photo- 
Table 1 RTA measurements (SD) of retinal thickness

\begin{tabular}{lllll}
\hline & $\begin{array}{l}\text { Normal eyes } \\
\text { (46 eyes) }\end{array}$ & $\begin{array}{l}\text { CSMO } \\
(21 \text { eyes })\end{array}$ & $\begin{array}{l}\text { "Dry" macula } \\
(10 \text { eyes })\end{array}$ & $\begin{array}{l}\text { CMO } \\
\text { (10 eyes) }\end{array}$ \\
\hline Foveal thickness $(\mu \mathrm{m})$ & $178(44)$ & $427(175)$ & $315(71)$ & $875(287)$ \\
Macular thickness $(\mu \mathrm{m})$ & $311(51)$ & $566(202)$ & $414(94)$ & \\
\hline
\end{tabular}

$\mathrm{CMO}=$ diabetic patients with cystoid macular oedema; $\mathrm{CSMO}=$ diabetic patients with clinically significant macular oedema; "Dry" macula $=$ diabetic macular oedema following laser treatment.

coagulation was between $200 \mu \mathrm{m}$ and $421 \mu \mathrm{m}$, with an average of 315 (71) $\mu \mathrm{m}$, a $77 \%$ increase compared with the normal value, and a $26 \%$ decrease in thickness compared with the CSMO eyes. The eyes with CMO displayed the largest thickening, which ranged between $470 \mu \mathrm{m}$ and $1230 \mu \mathrm{m}$, while the average was 875 (287) $\mu \mathrm{m}$ (a 390\% increase compared with normal values). The average thickness at 500 $\mu \mathrm{m}$ surrounding the fovea were as follows. The CSMO eyes had an average retinal thickness of $566(202) \mu \mathrm{m}$ (range $221-723 \mu \mathrm{m})$ compared with 311 (51) $\mu \mathrm{m}$ (range 250-388 $\mu \mathrm{m}$ ) in normal eyes, a $80 \%$ increase, whereas the dry macula group following laser treatment had an average thickness of 414 (94) $\mu \mathrm{m}$ (range 270-638 $\mu \mathrm{m}$ ), a $27 \%$ decrease compared with the CSMO eyes, and a $33 \%$ increase compared eyes of healthy controls. These relations persisted in subgroups divided by age as well (Table 2).

Statistically significant differences were found in central thickness between the four groups $(p=0.0001)$. All pairwise comparisons between the groups (controls, CSMO, treated "dry" foveas, and CMO) were also statistically significant $(p<0.05)$.

The differences in the averaged thickness of $500 \mu \mathrm{m}$ around the foveal centre between the three groups (controls, CSMO, and treated eyes) were statistically significant, with a $p$ value of 0.0001 . The pairwise comparisons were also statistically significant $(\mathrm{p}<0.05)$.

In the younger age group (20-34 years), the differences in central thickness between the groups (controls and CSMO) were statistically significant, with $p$ value of 0.0001 . The pairwise comparisons were also statistically significant $(p<0.05)$. The differences in the average thickness around the fovea between the groups (CSMO and controls) were statistically significant ( $p=0.0001)$, and the pairwise comparisons between controls and CSMO were also significant $(\mathrm{p}<0.05)$.

In the second age group (35-49 years), the differences in central and average thickness around the fovea were statistically significant $(\mathrm{p}=0.0001)$, the pairwise comparisons between the controls and the CSMO eyes were significant, whereas the comparisons between the controls and the "dry" treated eyes were non-significant $(p=0.05)$.

In the oldest age group (50-70 years), the differences in the central thickness were statistically significant $(p=0.0001)$, and the pairwise comparisons were significant between the CSMO group and the rest of the eyes, but were not significant between the normal eyes and the CSMO and treated eyes. The differences in the average thickness around the fovea were significant $(\mathrm{p}=0.02)$, and the pairwise comparisons were significant between the controls and the CSMO eyes and non-significant between the controls and the treated "dry" eyes.

\section{Discussion}

Previous investigators demonstrated the reliability of the delivery method of a green helium neon laser in a parallel beam of light and measurement of the retinal thickness in primates and in humans. ${ }^{12}$ These investigators measured the retinal thickness in patients with diabetic macular oedema and applied this method to quantitate macular thickness before and after focal treatment for macular oedema. ${ }^{78}$ The RTA $^{45}$ is an improvement of this method and was developed to enhance the visualisation of fine structures of the retina and to measure the thickness of a scanned area of 6 $\times 6 \mathrm{~mm}$. The green, helium neon laser illumination is ideally suited because of the narrowness of the beam, the enhanced brightness, and the monochromasy in green which reduces background scatter. These advantages allow for visualisation of fine retinal structures and measurement of the exact width of each point, as well as providing a quantitative profile of the retinal thickness of the whole scanned area.

The RTA (RetinaMap), a retinal thickness scanner provides the following advantages:

(1) replacement of the contact lens by indirect optics which do not necessitate contact with the examined eye;

(2) replacement of the film camera and digitalised instrumentation by an electronic camera which directly inputs the slit images acquired from the examined retina into the computer in real time;

(3) a scanner is included in the optical system which allows acquisition of 10 slit images spaced evenly over a $2 \mathrm{~mm}$ area on the retina within $200 \mathrm{~ms}$;

(4) operation and analysis software for fast and easy operation of the instrument.

We investigated the correlation between subjective clinical assessment of diabetic macular

Table 2 Macular thickness $(\mu \mathrm{m})$ measured by the RTA in age matched groups

\begin{tabular}{|c|c|c|c|c|c|c|c|c|}
\hline & \multicolumn{2}{|l|}{$<20$ years } & \multicolumn{2}{|l|}{ 20-34 years } & \multicolumn{2}{|l|}{$35-49$ years } & \multicolumn{2}{|l|}{$50-70$ years } \\
\hline & Fovea & Macula & Fovea & Macula & Fovea & Macula & Fovea & Macula \\
\hline Normal (SD) & $184(21)$ & $328(47)$ & $166(38)$ & $288(33)$ & $175(45)$ & $318(49)$ & $222(52)$ & $340(77)$ \\
\hline No of eyes & 5 & 5 & 6 & 6 & 30 & 30 & 5 & 5 \\
\hline CSMO (SD) & & & $491(202)$ & $691(253)$ & $352(142)$ & $499(96)$ & $410(171)$ & $542(175)$ \\
\hline No of eyes & & & 8 & 8 & 5 & 5 & 8 & 8 \\
\hline Dry (SD) & & & & & $239(44)$ & $317(55)$ & $347(53)$ & $455(74)$ \\
\hline No of eyes & & & & & 3 & 3 & 7 & 7 \\
\hline
\end{tabular}


oedema and quantitative measurements of the retinal thickness. We also measured the foveal thickness in a group of treated eyes judged clinically as having a "dry" macula for at least 4 months after treatment. Our results showed good correlation between the clinical diagnosis of macular oedema and the objective quantification of the macular thickness. The CMO retina exhibited the largest thickening, which constituted a $390 \%$ increase compared with normal values. The non-CMO oedematous macula had a $144 \%$ increase in thickness in the foveal centre compared with normal macula, and a $80 \%$ increase $500 \mu \mathrm{m}$ around the foveal centre. The retina which were judged as being "dry" after focal laser treatment had a $26 \%$ decrease in thickness compared with the CSMO in the centre of the fovea and $500 \mu \mathrm{m}$ around it, but still had a $77 \%$ increase compared with the normal values in the centre of the fovea and a 33\% increase compared with the normal values in a circle $500 \mu \mathrm{m}$ around the fovea. These preliminary results show that laser treatment is effective in reducing the macular thickening. They also highlight the fact that CMO constitutes a worse aspect of diabetic macular oedema compared with CSMO without cystoid changes. Its grim prognosis may be related to the degree of thickness which is more severe than in non-CMO diabetic macular oedema.

The reproducibility of the measurements was found to be plus or minus $10.6 \mu \mathrm{m}$ of the macular thickness. These measurements were taken in a group of healthy individuals. The mean retinal thickness in the scanned area of the normal macula corresponded to the results of others. ${ }^{49}$

The retinal thickness analysis of diabetic macular oedema and changes occurring following focal laser treatment observed by Shahidi $e \mathrm{al}^{78}$ also correspond to our results using a beam of green, helium neon laser projected on a selected narrow area of the retina.

The major advantage of the RTA is the option to scan a relatively wide area of the retinal tissue in a short acquisition time. This option is of great importance in the objective assessment of the efficacy of laser treatment in diabetic macular oedema or other therapeutic regimen, such as acetazolamide or antiinflammatory agents, used in CMO. Moreover, the operator can easily choose a selected point of interest in the fundus and analyse the intraretinal structures in cases in which a small retinal area is affected (such as macular hole or cyst).

This method of objective measurement of the retinal thickness in various macular diseases serves as a useful and sensitive tool for the diagnosis and follow up of diabetic macular oedema, as well as for the assessment of the efficacy of laser treatment of this condition.

1 Zeimer RC, Shahidi M, Mori M, et al. In vivo evaluation of a noninvasive method to measure the retinal thickness in primates. Arch Ophthalmol 1989;107:1006-9.

2 Shahidi M, Zeimer R, Mori M. Topography of the retinal thickness in normal subjects. Ophthalmology 1990;97: 1120-4.

3 Mori MT, Shahidi M, Zeimer RC. New noninvasive method to measure changes in the nerve fiber layer thickness, methodology and reproducibility in normal subjects. Invest Ophthalmol Vis Sci (Suppl) 1989;30:175.

4 Zeimer R, Shahidi M, Mori M, et al. A new method for rapid mapping of the retinal thickness at the posterior pole. Invest mapping of the retinal thickness at the post

5 Landau D, Schneidman EM, Jacobovitz T, et al. Quantitative in vivo retinal thickness measurements in healthy subjects. Ophthalmology 1997;104:639-42.

6 ETDRS Report Number 7. Early treatment diabetic retinopathy study design and baseline patient characteristics. Ophthalmology 1991;98:741-56.

7 Shahidi M, Ogura Y, Blair NP, et al. Retinal thickness analysis for quantitative assessment of diabetic macular edema. Arch Ophthalmol 1991;109:1115-9.

8 Shahidi M, Ogura Y, Blair NP, et al. Retinal thickness change after focal laser treatment of diabetic macula oedema. Br f Ophthalmol 1994;78:827-30.

9 Arsani S, Zeimer R, Goldberg MF, et al. Application of rapid scanning retinal thickness analysis in retinal diseases. Ophthalmology 1997;104:1145-51. 